\title{
Comparison of airtraq and trueview EVO2 with macintosh laryngoscope for endotracheal intubation by experienced anaesthesiologists: a controlled clinical trial
}

\begin{abstract}
Background: The purpose of the study was to evaluate the efficacy of two new laryngoscopes by experienced anaesthesiologists. Airtraq and Truview EVO2 were compared with gold standard Macintosh laryngoscope for endotracheal intubation of elective surgical patients with normal airway distribution.

Methods: In prospective, randomised and controlled manner 150 patients of either sex above 18 yrs, presenting for elective surgery were randomly allocated in three equal groups. Endotracheal intubation was done using either Macintosh (group ML), Airtraq (group AQ) or Trueview (groupTL) laryngoscope. Laryngoscopic view of glottis (Cormac and Lehane grade), time taken for intubation, subjective assessment for ease of intubation, intubation difficulty scale (IDS) score as well as attempts for successful intubation, success rate and soft tissue injury during laryngoscopy and intubation were compared.

Results: All the patients intubated with Airtraq and Trueview had Cormack and Lehane grade I or II and none had grade III in comparison with $42(84 \%)$ having grade I or II and $8(16 \%)$ having grade III $(\mathrm{p}<0.05)$. Mean time taken for intubation was significantly longer with Truview $(35.3 \pm 11.5 \mathrm{sec})$ and comparable between Airtraq $(19.2 \pm 4.7 \mathrm{sec})$ and Macintosh $(24.4 \pm 13.8 \mathrm{sec})(\mathrm{p}<0.05)$. On subjective assessment Truview was rated as most difficult device to use for intubation amongst three laryngoscopes. Truview and Airtraq had mean IDS scores of $<1(0.75 \pm 0.4$ and $0.56 \pm 0.5$ respectively $)$ while a higher score $(1.76 \pm 2)$ was found with Macintosh $(\mathrm{p}<0.05)$. No significant difference was noted in terms of attempts at intubation, success rate and soft tissue injury.

Conclusion: We concluded that Airtraq was a promising and reliable alternative to Macintosh for endotracheal intubation of patients with normal airway characteristics in experienced hands. Although True view provided better intubating conditions than Macintosh, it took longer time for intubation and on subjective assessment, it was graded as difficult to use.
\end{abstract}

Keywords: tracheal intubation, macintosh laryngoscope, truview evo2 laryngoscope, airtraq
Volume 3 Issue 4 - 2015

\author{
Yogita Dwivedi, 'Vinay Shukla, ${ }^{2}$ Uma \\ Srivastava, ${ }^{3}$ Arpita Saxena, I Amrita Gupta, I \\ Ramya Mishra, ${ }^{2}$ Tapas Kumar Singh ${ }^{2}$ \\ 'Assistant Professor, Department of Anaesthesiology and \\ Critical Care, India \\ ${ }^{2}$ Senior Resident, Department of Anaesthesiology and Critical \\ Care, India \\ 3Professor, Department of Anaesthesiology and Critical Care, \\ India
}

Correspondence: Uma Srivastava, Department of Anaesthesiology and Critical Care, Sarojini Naidu Medical College, flat no 2b2, bagla kunj appt, Agra, Uttar Pradesh, INDIA. 282003, Agra, India, Tel +91562252207l,

Email drumasrivastava@gmail.com

Received: October 21, 2015 | Published: November 16, 2015

\section{Introduction}

Laryngoscopy and tracheal intubation require lots of clinical experience to master and is a core skill for all anaesthetists. Despite use of Macintosh laryngoscope for decades, failure to successfully intubate the patient remains the leading cause of morbidity and mortality in operating rooms. Consequently a number of other devices that facilitate endotracheal intubation with low failure rate have been added to the armamentarium of the anaesthesiologists which may replace the conventional direct laryngoscopy. Previous manikin trial indicate towards better intubating conditions with most of the new indirect optical laryngoscopes when compared to direct laryngoscopy using Macintosh. But, the results of these studies cannot be extrapolated to clinical scenarios due to use of rigid plastics, the lack of collapsible soft tissues and absence of secretions in the manikin.

Airtraq (Prodol Meditec, Vizcaya, Spain, 2005) is a novel optical laryngoscope with series of lenses, mirrors and an exaggerated blade curvature which provides glottis display without any deviation in normal position of oral, pharyngeal or tracheal axes and allows intubation with minimal manipulation of neck. The tracheal tube does not obstruct the view during intubation. ${ }^{2}$ Truview EVO2 laryngoscope
(Truphatek International ${ }^{\circledR}$, Israel) provides unmagnified anterior refraction of 42 degrees in the line of sight with minimal manipulation of the head, neck, instrument or soft tissue. ${ }^{3}$

Based on previous clinical, ${ }^{3,4}$ manikin ${ }^{1}$ trials and meta-analysis, ${ }^{2}$ we hypothesized that Airtraq and Truview Laryngoscope would perform better for airway management of surgical patients with normal airway data. The present study was planned to evaluate the usefulness of Airtraq and Truview by experienced users in patients with low risk of difficult airway. The new devices were compared with conventional Macintosh laryngoscope. The primary outcome measures were Cormack and Lehane grade and intubation difficulty scale (IDS) score whereas secondary measures were time to successfully intubate and success rate, subjective difficulty in intubation and complications with each device.

\section{Material \& methods}

The study was conducted after approval from Institutional Review Board and written and informed consent of patients. 150 ASA physical status I-II patients, aged 18 years of age or older, of either sex, scheduled for elective surgical procedures requiring tracheal 
intubation were recruited in this prospective randomized study. Exclusion criteria were patients with risk of pulmonary aspiration, undergoing emergency surgery and those with anticipated difficult airway (Mallapatti grade IV, thyromental distance $<6 \mathrm{~cm}$, mouth opening $<1.5 \mathrm{~cm}$, body mask index $>35 \mathrm{~kg}-\mathrm{m}$ ). Preoperative airway evaluation was performed by an anaesthetist a day prior to surgery, who was not involved in subsequent anaesthetic management.

Eight hours of fasting was advised and premedication with oral alprazolam $0.25-0.5 \mathrm{mg}$ was given a night before surgery and in the morning of surgery. After shifting the patient to OR, intravenous access was secured and monitors including $\mathrm{SpO}$, NIBP, ECG, capnography were applied and baseline parameters recorded. All patients received a standardized general anaesthetic. Prior to induction all patients were premedicated with glycopyrrolate $0.2 \mathrm{mg}$, midazolam $1 \mathrm{mg}$, fentanyl $2 \mathrm{micro}$ grams $/ \mathrm{kg}$. After preoxygenation, sleep dose of propofol $2-3 \mathrm{mg} / \mathrm{kg}$ was titrated to induce anaesthesia followed by vecuronium $0.1 \mathrm{mg} / \mathrm{kg}$ intravenously. Three minutes after vecuronium trachea was intubated (after confirming adequacy of neuromuscular block by peripheral nerve stimulator) with a 7.5 gauge ETT in females and 8.5 gauge in males using the laryngoscope depending upon group allocation. The allocation sequence was generated by chit in box system opened only after patients consent was obtained. 150 chits with initials of ML, AQ, TL were labelled and picked up to ensure equal number of patients in each group. The patients trachea was intubated with either Macintosh (size 3 or 4 blade, Group ML), Airtraq ( 3 or 4 size, Group AQ), or Truview EVo2, (Group TL) by one of the two anaesthesiologist who were well experienced with use of new laryngoscopes (100 intubations in manikin \& 50 in patients).

Airtraq was loaded with endotracheal tube, held in left hand and passed into the mouth over tongue in midline to place its tip in the valleculla. Once the glottis was in the centre of the view seen from the viewfinder, the ETT was then passed from its position in the channel through the vocal cords under vision. For Airtraq activation time of 30 to 60 seconds was required to warm up the lens to reduce fogging. ${ }^{2}$ Truview EVO2 scope was held in left hand and advanced in mouth until epiglottis was visible. After viewing the vocal cords through eyepiece, eyes were taken off the eyepiece while holding instrument steady. To reduce fogging of distal lens insufflations of oxygen (8-10 $\mathrm{L} / \mathrm{min}$.) from side port was used. Correct intubation was confirmed in each patient by auscultation and by presence of $\mathrm{CO}_{2}$ in exhaled breath. The primary end points were Cormac and Lehane grade for glottis visualization and Intubation Difficulty scale (IDS) score developed by Adnet et al. ${ }^{5}$ (Table 1). The view of glottis at laryngoscopy was scored according to Cormack and Lehane grading after primary visualization of glottis. Laryngeal manipulation to improve the laryngoscopic view for intubation was done if needed. Time taken for intubation (time from introduction of laryngoscope blade into the patient's mouth until capnographic trace of $\mathrm{CO}_{2}$ obtained in exhaled air), success rate of intubation and number of attempts required for successful intubation were recorded. If intubation was not successful after three attempts or time taken for intubation was more than 120 seconds, it was assigned as failed and intubation was carried by alternate laryngoscope. A semirigid stylet was used to assist intubation if needed during laryngoscopy with Macintosh laryngoscope. After completion of intubation the anaesthesiologist judged ease of intubation on the subjective basis as easy, difficult or very difficult and also recorded Intubation Difficulty scale (IDS) score based on variables given in Table 1.

\section{Statistical analysis}

We based our sample size estimation on our primary outcome measures namely IDS score and Cormac and Lehane grading. On the basis of a pilot study of 45 patients we considered that a reduction in mean IDS score of 1.5 and standard deviation of 2 from Macintosh group would be clinically important. Using $\alpha=0.05$ and $\beta=0.2$, we estimated that minmum 50 patients would be required in each group. Patient's demographic, airway assessment data, Cormack and Lehane grade, number of attempts for intubation and anaesthetist evaluation of difficulty were analysed by chi square or paired t-test as appropriate. For comparison of IDS scoring paired t test was used. Time taken for intubation was analysed using analysis of variance (ANOVA). Each device was compared with both of the other devices in these post hoc tests. Data are presented as mean (SD), with categorical data presented as number (\%) and as frequencies. $\mathrm{P}$ value $<0.05$ was considered as significant. We used SPSS Software version 13 and Statpages .org for analyses.

\section{Results}

There were no differences in demographic and airway variables (Table 2). Total 150 patients were recruited and there was no failure in intubation in any group. Overall success rate for intubation was not different among the three devices (Table 3 ) although first attempt success rate was higher in AQ and ML groups. However, a greater number of optimization manoeuvres were required to facilitate tracheal intubation in ML group compared to other two groups (Table 3). Grade I Cormack and Lehane glottis view was obtained in $45(90 \%)$ and $43(86 \%)$ of patients intubated with Airtraq and Truview laryngoscopes respectively compared to $35(70 \%)$ with Macintosh. In ML group a greater percentage of patients had Cormack and Lehane grade III (16\%) in comparison with none in AQ and TL groups (Table 3) $(\mathrm{p}<0.05)$.

Time taken for intubation (TTI) was longest in patients intubated with Truview $(35.3 \pm 11.5 \mathrm{sec})$ followed by those intubated with Macintosh $(24.4 \pm 13.8 \mathrm{sec})$ and Airtraq $(19.2 \pm 4.7 \mathrm{sec})$ (Table 3). On statistical comparison TTI was significantly longer in group TL compared with groups AQ and ML $(\mathrm{p}<0.05)$. While assessing the ease of intubation by subjective evaluation, anaesthetists found intubation as easy in 78,71 and $33 \%$ of patients in groups AQ, ML and TL respectively $(\mathrm{p}<0.01)$. Mean IDS score was $0.56 \pm 0.4$ in group $\mathrm{AQ}, 0.75 \pm 0.5$ in group TL and $1.76 \pm 2$ in group $\mathrm{ML}(\mathrm{p}<0.05)$. Significantly more patients in study groups AQ and TL had IDS score of $<1$ compared to group ML (Table 3 ). There were no betweengroup differences in the incidence of complications including minor laceration of lips or blood on laryngoscope blade or dental trauma. Arterial desaturation was not seen in any patient.

\section{Discussion}

Inspite of introduction of variety of laryngoscopes, Macintosh laryngoscope still remains the most frequently used intubation aid although it requires alignment of various axes which is a difficult skill to learn, acquire ${ }^{6}$ and maintain. ${ }^{7}$ Recent alternatives to Macintosh laryngoscope provide magnified/anterior refracted view of glottis without need for alignment of axis. We evaluated newer devices against present 'gold standard' as recommended by Cook. ${ }^{8}$ Experienced anaesthesiologists conducted the trial in patients with low risk of difficult intubation because new devices require expertise in such patients before use in high risk patients.

Our study demonstrated that overall success rate of intubation was high in all the three groups but first attempt success rate was significantly higher in patients intubated with Airtraq or Macintosh. TTI was shortest in group AQ and longest in group TL with that in group ML lying between these two. While overall IDS scores were 
lower in all the groups, both mean score and number of patients with IDS score of $\geq 1$ were very few in study groups AQ and TL in comparison to group ML. On laryngoscopy, Cormac and Lehane grade was 1 or 2 in most patients in each group with no patient of grade III in groups AQ and TL compared to three in group ML. Optimization manoeuvres were more frequently required in group ML. incidence of dental trauma or soft tissue injury was infrequently seen in all the groups.

Table I Intubation Difficulty Scale (Ids) Score.

\begin{tabular}{ll}
\hline Parameter & Score \\
\hline Number $(\mathrm{n})$ of attempts at intubation & $\mathrm{n}-\mathrm{I}$ \\
Number $(\mathrm{n})$ of operators attempting intubation & $\mathrm{n}-\mathrm{I}$ \\
Number of alternative intubation techniques & $\mathrm{n}-\mathrm{I}$ \\
Cormack and Lehane grade & Grade-I \\
Lifting force required at laryngoscopy & $0=$ normal, I = increased \\
Necessity for External laryngeal pressure & $0=$ not applied, I = applied \\
Position of vocal cords at intubation & 0 -abduction/visualized, I-adduction \\
\hline
\end{tabular}

IDS=Sum of all 7 parameters [IDS=0 (Easy intubation), IDS=I-5 (moderate difficulty), IDS=6-I5 ( very difficult to impossible)].

Table 2 Demographic and Airway Data

\begin{tabular}{llll}
\hline Variable & Group-AQ & Group-TL & Group-ML \\
\cline { 2 - 4 } Age $(\mathrm{yrs})$ & 38.8 & $\mathrm{n}=50$ & $\mathrm{n}=50$ \\
Mean (SD) & -10.91 & 36.33 & 39.55 \\
Height $(\mathrm{mts})$ & 1.63 & -11.79 & -13.03 \\
Mean $(\mathrm{SD})$ & -0.1 & 1.62 & 1.63 \\
Weight $(\mathrm{kg})$ & 63.38 & -0.08 & -0.09 \\
Mean $(\mathrm{SD})$ & -10.31 & 63.06 & 62.41 \\
Sex M/F & $30 / 20$ & -9.88 & -10.19 \\
Body mass index $\left(\mathrm{kgm}^{-2}\right)$ & $28.2 \pm 3.7$ & $29 / 21$ & $28 / 22$ \\
Mouth Opening $(\mathrm{cm})$ & $4.38 \pm 0.26$ & $27.8 \pm 4.7$ & $27.7 \pm 6.4$ \\
Thyromental Distance $(\mathrm{cm})$ & $6.92 \pm 0.24$ & $6.99 \pm 0.19$ & $4.33 \pm 0.28$ \\
Mallampatti grade $\mathrm{I} / 2 / 3 / 4$ & $24 / 17 / 9 / 0$ & $25 / 18 / 7 / 0$ & $6.98 \pm 0.14$ \\
\end{tabular}

Table 3 Study Data for Intubation Attempts.

\begin{tabular}{|c|c|c|c|}
\hline Parameter assessed & Group AQ & Group TL & Group ML \\
\hline IDS Score (Mean \pm SD) & $0.56 \pm 0.4 *$ & $0.75 \pm 0.5^{*}$ & $1.76 \pm 2$ \\
\hline \multicolumn{4}{|l|}{ IDS Score (no \& \%) } \\
\hline 0 & $42(84 \%)$ & $39(78 \%)$ & $26(52 \%)$ \\
\hline I & $8(16 \%)$ & $9(18 \%)$ & $14(28 \%)$ \\
\hline$>1$ & $0(0 \%)$ & $2(4 \%)$ & $10(20 \%)$ \\
\hline Cormack \& Lehane grade ( $1 / 2 / 3)$ & $45 / 5 / 0 *$ & $43 / 7 / 0 *$ & $35 / 7 / 8$ \\
\hline Optimization manoeuvres(No.) 0/I/2 & $46 / 4 / 0^{*}$ & $43 / 6 / I^{*}$ & $34 / 10 / 6$ \\
\hline Time taken for intubation (Sec) ( Mean \pm SD) & $19.2 \pm 4.8 * *$ & $35.3 \pm 11.5$ & $24.4 \pm 13.9 * *$ \\
\hline Subjective evaluation of intubation( Easy/Difficult/Very difficult) & $39 / 10 / 1 * *$ & $16 / 22 / 12$ & $36 / 11 / 3 * *$ \\
\hline Overall success rate & $100 \%$ & $100 \%$ & $100 \%$ \\
\hline Number of attempts I/2/3/failure & $42 / 8 / 0 / 0$ & $36 / 12 / 2 / 0$ & $34 / 9 / 7 / 0$ \\
\hline
\end{tabular}

*significant in relation to group $\mathrm{ML}$

**significant in relation to group TL

The view obtained on laryngoscopy is a major factor in determining difficulty of intubation. ${ }^{9}$ Many clinical and manikin trials conducted till date have taken glottic view as the most important parameter for comparing new laryngoscopic devices. ${ }^{3,5,9} \mathrm{~A}$ unique combination of an extremely curved blade and an inbuilt optical system enables Airtraq to provide a panoramic view of glottis making laryngoscopy easy. ${ }^{10}$ Similarly, a 42 degree anterior refracted glottic view with Trueview reduces the difficulties encountered during direct laryngoscopy. This may be a reason why a greater number of patients intubated with Airtraq and Truview had Cormack and Lehane grade I (45/50 and 43/50 in groups AQ and TL respectively) and none had grade III. Whilst those intubated with Macintosh 35/50 patients had Cormac and Lehane grade I and eight patients grade III. Other studies also reported similar results. ${ }^{11,12}$ More patients (16/50) required additional manoeuvres to improve glottis view in group ML than other groups. Improvement in Cormack and Lehane grade at least by one has been reported during both routine and difficult airway scenarios ${ }^{13-16}$ while laryngoscopy with Airtraq and Trueview laryngoscopes.

Although Cormac Lehane grading is the most popular means of describing laryngeal view, it should be borne in mind that this system is devised for direct laryngoscopy therefore it can under estimate the difficulty of intubation with indirect laryngoscopes. ${ }^{17}$ As also reported by Maharaj et al. ${ }^{11}$ this grading system provided a useful comparison of laryngoscopic view of glottis in this study. POGO score, is a more refined way to categorize laryngeal view and also has better interphysician reliability than Cormac and Lehane grading. ${ }^{18}$ 
The intubation difficulty scale (IDS) score is a quantitative scale incorporating multiple indices of intubation difficulty (Table 1) that more objectively quantify the complexity of tracheal intubation. It is a blend of subjective and objective criteria. An IDS score of 0-1 represents ideal intubating conditions. The overall IDS scores were lower in all the three groups as would be expected in this population of patients with low risk of intubation difficulty. Both mean IDS score and number of patients with an IDS score $\geq 1$ were significantly lower in AQ and TL groups compared to ML group. Lower IDS scores in patients intubated with newer laryngoscopes can be attributed to better layngoscopic view, less optimization manoeuvres and lifting force required in comparison to Macintosh as reported previously., $5,13,19-21$

The mean time taken for intubation and related morbidity make them important parameters to be studied for assessing the efficacy of new laryngoscopic devices. Time taken for intubation (TTI) was significantly longer with Truview in comparison with the other two laryngoscopes. In concurrence with our results, previous studies comparing Trueview with Macintosh have reported that time taken for intubation was more with former laryngoscope irrespective of airway anatomy and grading of airway difficulty. ${ }^{3,19}$ Contradictory to this, Chalkiedes et al. ${ }^{16}$ found intubation time longer with Airtraq (29.6 \pm $8.5 \mathrm{sec}$. $)$ in comparison to Macintosh $(23.7 \pm 5.9$ seconds $)$.

The prolonged intubation time with Truview could possibly be due to considerable difficulty in advancing the endotracheal tube towards the glottis and also due to lack of practice with the new device. ${ }^{6,19,20}$ We opine that this difference of few seconds in intubation time is of no clinical relevance in elective patients but may raise concern in emergency situations. A meta analysis ${ }^{2}$ reviewing 1000 patients concluded that the mean time taken for intubation with Airtraq was reduced by 14-16 seconds in patients with normal airway and by $22-25$ seconds with difficult airway in comparison to Macintosh laryngoscope.

We also subjectively rated the newer intubation devices as easier to use in comparison with conventional and more familiar Macintosh laryngoscope. On subjective assessment anaesthesiologist rated Truview as difficult device to use for intubation in most patients. Subjective difficulty in using Truview observed by us and supported by previous studies ${ }^{3,20,21}$ can be attributed to the peculiar technique during use of laryngoscope and also camera mounted may feel heavy to operate ${ }^{21}$ and need of good hand eye coordination. We assume that the problems related to the intubation can be overcome considerably by more frequent use and practice with the device as evidenced in this study that intubation time consistently reduced as the study advanced.

Macintosh laryngoscope requires steerage of endotracheal tube which is difficult skill to learn in initial stages. Airtraq has a prefixed channel to align the tube towards glottis. In some patients while using Airtraq endotracheal tube struck posteriorly. Attempts to withdraw the Airtraq slightly upward with tube in situ or using a stylet which reduced the distance between the tip of endotracheal tube and scope, ${ }^{22}$ helped to overcome the above problem. Success rate of intubation was more than $95 \%$ in each group although first attempt success rate was higher in AQ and ML group. ${ }^{12,13}$ All three laryngoscopes performed equally well in experienced hands.

Direct dental trauma and injury to oral tissue has been reported in $6.9 \%$ of patients during direct laryngoscopy with conventional laryngoscope. ${ }^{23}$ Previous manikin study ${ }^{23}$ reported less potential for trauma to teeth and soft tissues with newer airway devices. In this study the incidence of trauma to lip, teeth and upper airway was almost nil.
There are important limitations with regard to our study. First, we acknowledge that the potential for bias exists, as it is impossible to blind the anaesthetist to the device being used. Certain measurements used in this study, such as laryngoscopic grading, are by their nature subjective. Despite this, Cormac and Lehane grading has been used widely in clinical practice, the appropriateness of using this system with indirect laryngoscopy is open to question. We recruited patients with normal airway characteristics, therefore our results cannot be extrapolated for patients with difficult airway. Furthermore, our study was not a crossover trial so we cannot comment on improvement of Cormack and Lehane grade with the new laryngoscopic devices but our study proves that Airtraq and Truview show better laryngoscopic view in comparison to Macintosh. Lastly, the laryngoscopes were used by experienced anaesthetists, so results may not be similar for less experienced users.

\section{Conclusion}

On the basis of our observations we conclude that Airtraq and Truview laryngoscopes improved Cormack and Lehane grade and had lower IDS scores in comparison to Macintosh. Airtraq was easy to use and needed less time to intubate as with Macintosh compared to Trueview which was a bit difficult to handle and also needed longer time to intubate. Airtraq proved to be a useful device for endotracheal intubation of patients with normal airway in experienced hands but being single use, cost can be a limiting factor in developing countries. Further studies are required for use by novices and less experienced users with substantial number of patients with both easy and difficult airway to strengthen current study.

\section{Conflicts of Interest}

The authors do not have any Conflict of interests.

\section{Acknowledgments}

None.

\section{Funding}

None.

\section{References}

1. Maharaj $\mathrm{CH}$, Higgins BD, Harte $\mathrm{BH}$, et al. Evaluation of intubation using the Airtraq or Macintosh laryngoscope by anaesthetists in easy and simulated difficult laryngoscopy-a manikin study. Anaesthesia. 2006;61(5):469-447.

2. Lu Y, Jiang H, Zhu Y S. Airtraq laryngoscope versus conventional Macintosh: A systematic review and meta-analysis. Anaesthesia. 2001;66:1160-1167.

3. Saxena A, Madan M, Srivastava U, et al. Role of Truview EVO2 Laryngoscope in airway management of elective surgical patients : A comparison with Macintosh laryngoscope. Ind $J$ Anaesth. 2013;57(3):276-281.

4. Maharaj $\mathrm{CH}$, Buckley E, Harte BH, et al. Endotracheal intubation in patients with cervical spine immobilization: A comparison of Macintosh and AirtraqTM laryngoscopes. Anesthesiology. 2007;107(1):53-59.

5. AdnetF, Borron SW, Racine SX, etal. The intubation difficulty scale(IDS): proposal and evaluation of a new score characterizing the complexity of endotracheal intubation. Anesthesiology. 1997;87(6):1290-1297.

6. Wang HE, Seitz SR, Hostler D, et al. Defining the learning curve for paramedic student endotracheal intubation. Prehosp Emerg Care. 2005;9(2):156-162. 
7. Garza AG, Gratton MC, Coontz D, et al. Effect of paramedic experience on orotracheal intubation success rates. J Emerg Med. 2003;25(3):251256.

8. Cook TM. Novel airway devices: spoilt for choice? Anaesthesia. 2003;58(2):107-110.

9. Peterson GN, Domino KB, Caplan RA, et al. (Management of the difficult airway : a close claim analysis. Anesthesiology. 2005;103(1): 33-39.

10. Ali QE, Amir SH, Siddiqui OA, et al. Airtraq ${ }^{\circledR}$ Optical laryngoscope for tracheal intubation in patients with severe ankylosing spondylitis: A report of two cases. Ind J Anaesth. 2012;56(2):165-167.

11. Maharaj CH, O’Croinin D, Curley G, et al. A comparison of tracheal intubation using Airtraq or Macintosh laryngoscope in routine airway management: a randomized controlled clinical trial. Anaesthesia. 2006;61(11):1093-1099.

12. McElwain J, Laffey JG. Comparison of the C-MAC, Airtraq, and Macintosh laryngoscopes in patients undergoing tracheal intubation with cervical spine immobilization. Br J Anaesth. 2011;107:258-264.

13. Maharaj CH, Costello SF, Higgins BD, et al. Learning \& performance of tracheal intubation by novice personnel: a comparison of the Airtraq and Macintosh Laryngoscope. Anaesthesia. 2006;61(7):671-677.

14. Bhalla S,Bhola $\mathrm{R}$, Gupta $\mathrm{R}$, et al. Tracheal intubation in patients with cervical spine immobilization: a comparison of McGrath videolaryngoscope and Trueview laryngoscope. Ind $J$ Anaesth. 2014;58(3):269-274.

15. Singh I, Khaund A, Gupta A. Evaluation of Truview Ev02 laryngoscope in anticipateddifficult intubation- A comparison to Macintosh Laryngoscope. Ind J Anaesth. 2009;53(2):164-168.
16. Chalkeidis O, Kotsovolis G, Kalakonas A, et al. A comparison between the Airtraq and Macintosh laryngoscopes for routine airway management by experienced anesthesiologists: a randomized clinical trial. Acta Anaesthesiol Taiwan. 2010;48(1):15-20.

17. Ochroch EA, Hollander JE, Kush S, et al. Assesment of laryngeal view in direct laryngoscopy: The percentage of glottis opening (POGO) compared to Cormack and Lehane grading. Can J Anesth. 1999;46(10):987-990.

18. Khan RM, Maroo FM, Jain S. Truview Evo2 vs Macintosh laryngoscopy: Study of cardiovascular response and POGO scoring. J Anaesth Clin Pharm. 2008;24:311-314

19. Li JB, Xiong YC, Wong XL, et al. An Evaluation of Truview laryngoscope. Anaesthesia. 2007;62:940-943.

20. Barak M, Phillipchuck P, Abecassia P, et al. Comparison of Truview blade with Macintosh blade in adult patient. Anaesthesia. 2007;62(8):827833.

21. Malik MA, Maharaj CH, Harte BH, et al. Comparison of Macintosh, Truview Evo-2, Glidescope and airwayscope laryngoscope use in patients with cervical spine immobilization. $\mathrm{Br} J$ Anaesth. 2008;101(5):723-730.

22. Xue FS, He N, Liu JH, et al. A simple maneuver to facilitate tracheal intubation using the Airtraq laryngoscope with a reinforced endotracheal tube. Can J Anaesth. 2010;57(3):278-279.

23. Fung BK, Chan MY. Incidence of oral tissue trauma after the administration of general anaesthesia. Acta Anaesthesiologica Sinica. 2001;39(4):163-167. 